\title{
Determination of Sugar Content in Commercial Fruit Juices by Refractometric, Volumetric and Chromatographic Methods
}

\author{
Francesca Buiarellia, ${ }^{\mathrm{a}}$, Patrizia Di Filippo ${ }^{\mathrm{a}, \mathrm{b}}$, Donatella Pomata ${ }^{\mathrm{a}, \mathrm{b}}$, Carmela Riccardi ${ }^{\mathrm{a}, \mathrm{b}}$ and \\ Daniela Rago $^{\mathrm{a}}$
}

\author{
${ }^{a}$ Department of Chemistry, University of Rome "Sapienza", P.le Aldo Moro, 5-00185 Rome, Italy \\ ${ }^{b}$ Inail, Research, Certification and Control Division, DIPIA, Via di Fontana Candida, 1-00040 Rome, Italy
}

\begin{abstract}
In this paper several approaches are discussed for the direct analysis of the main sugars in different fruit juices. Refractometry, thin layer chromatography, volumetric analysis and high performance liquid chromatography with refractive index detector were tested and the results compared, discussing the advantages and disadvantages of each of them. Whereas the first method gives generically indications on the whole content of sugar and it doesn't require any prior manipulation of the sample, thin layer chromatography is useful only for qualitative purpose, on the other hand the third method, after removal of interferences, makes possible the determination of the reducing and not reducing sugar, and the last one allows the qualitative and quantitative determination of the saccharides singularly. It's very important to have not only knowledge about the chemical analysis of carbohydrates and their physicochemical properties, but especially how the methods can be used in product development for benefit of the public.
\end{abstract}

In the wide range of options for the determination of the mono and disaccharides in beverages, the approach selected must be robust, accurate, powerful and reproducible.

Keyword: Sugar composition, fruit juice, high performance liquid chromatography, lane-Eynon analysis.

\section{INTRODUCTION}

Carbohydrates are important food and beverage components affecting taste and nutrition and their determination is one of the most frequently used parameters in the food analysis, having considerable application in nutritional and biochemical studies. The qualitative/quantitative analysis of carbohydrates in food matrices is important for the energy evaluation, the nutritional labeling, the quality control and for identifying possible adulterations $[1,2]$. The food and beverage industry requires robust and reliable methods of analysis for simple sugars such as carbohydrates [3].

Most approved methods for sugar determination are indirect physical, enzymatic or semi-empirical chemical methods [4]. The Lane-Eynon General Volumetric Method (AOAC 923.09) is a titration method of determining the concentration of reducing sugars in a sample [5]. On the other hand, because of the high specificity and of the capability for simultaneous determination of several sugars, high performance liquid chromatography (HPLC) and gas-liquid chromatography (GLC) are advantageous and have been actively developed for the past decades. GC and GC-MS are excellent techniques for the analysis of carbohydrates; nevertheless the preparation of

*Address correspondence to this author at the Department of Chemistry, University of Rome "Sapienza", P.le Aldo Moro, 5-00185 Rome, Italy;

Tel: 39-6-49913645; Fax: 39-6-490631;

E-mail: francesca.buiarelli@uniroma1.it adequate derivatives is necessary [6,7], so HPLC is considered the more advantageous method for routine analysis and has become the preferred method for quantitating simple sugars in food and beverages. It is the most appropriate technique for accuracy, precision and practicability to the nutritional labeling purposes. HPLC often offers direct injection of a sample with little pretreatment and simple interpretation of chromatograms. Simple carbohydrates are highly polar, uncharged, and as they lack a chromophore cannot be measured directly by UV absorbance detectors, unless derivatized. The sugars can be determined in other matrices, different from food, using a great variety of chromatographic modes coupled to the refractive index detector (RID), light scattering (ELSD) or pulsed amperometric (PAD) detection and mass spectrometry [8-12]. Although a wide range of analytical techniques is available for mono and disaccharides in beverages, usually they are used singularly and there are few studies examining and comparing each other on the same samples. The variety of food and beverage products combined with raised expectations of quality and consistency of the consumer, has created a need for robust analytical methods that provide specific and reliable data on the composition of the food product.

With the aim of providing powerful and versatile analytical methods allowing for the selective and adequate determination of carbohydrate on beverages, in the present paper we selected four different analytical approaches to be tested in parallel on the same commercial fruit juices, commonly consumed. 
The investigated analytical methods considered were refractometric, volumetric and chromatographic (thin layer chromatography and HPLC). The purpose was to give serious consideration to the possible determinations that may be required before deciding on the analytical strategy, selecting the better one.

On the other hand, since an excessive sugar consumption is an ongoing concern, it is very important validating the claim of "no sugar added" appearing on some fruit marketed juices. So, we also compared the level of sugar content in freshly-extracted fruit juices and $100 \%$ fruit juices with a "no sugar added" label. The goal was to address the potential concern that commercially- $100 \%$ fruit juice with "no sugar added" may contain higher content of sugar compared to the extracted juice of fresh fruit. It is wishful that the method could be extended to a greater number of samples before assuming rough and hasty conclusions.

\section{EXPERIMENTAL}

\section{Chemicals, Reagents and Materials}

All the chemical and chromatographic reagents used were HPLC or analytical grade.

HPLC RS-Plus Methanol, isopropyl alcohol, ethyl acetate, ultra gradient Acetonitrile (ACN) were purchased by Carlo Erba Reagents, (Milan, Italy).
Ultra-pure water was produced with a Pure LabTM system (USF Elga, Ransbach-Baumbach, Germany).

Anhydrous analytical grade d-glucose (G), dfructose $(F)$ and sucrose (S), whose structures are shown in Figure 1, calcium carbonate $\mathrm{CaCO}_{3}$, chlorine acid $\mathrm{HCl} 37 \%$, sulfuric acid (96\%), glacial acetic acid, anisaldehyde, sodium hydroxide $\mathrm{NaOH}$ (98\%); Fehling's solution A e Fehling's solution B, Carrez I e Carrez II solutions, methylene blue indicator were purchased by Carlo Erba (Milan Italy).

Stock standard solutions of analytes were prepared by dissolving each compound in water $(100 \mathrm{mg} / \mathrm{mL})$ and storing them at $-20^{\circ} \mathrm{C}$ in the dark. Working solutions were prepared by successive dilution of the stock standard solutions.

Nylon filter $0,45 \mu \mathrm{m}$ was from Milleso $\AA-H N$ (Millipore,Usa); syringes (10-100 $\mu \mathrm{l}$ ) were from SGE (Chebios), homogenizer was from Ultraturax T18 (ICA), whereas the hand refractometer was purchased from Atago.

\section{Samples}

Based on the product popularity, we selected commercial brands of three different fruit juice: A) puree and juice of apricot with addition of sugar (syrup of glucose-fructose), B) Orange concentrated juice with

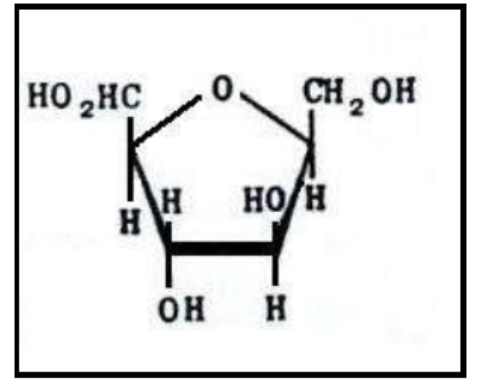

A) fructose

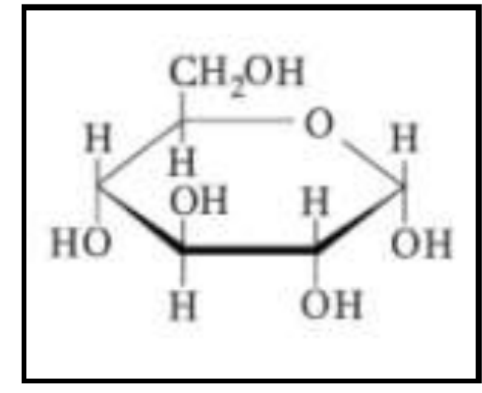

B) glucose

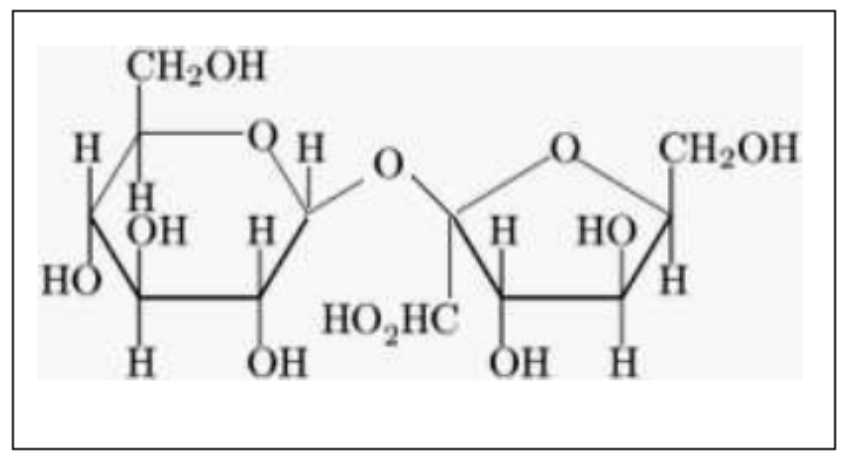

C) sucrose

Figure 1: Structures of the investigated compounds. 
no added sugar, C) concentrated juice of pineapple with no added sugar. Three samples of each drink were analysed. Pineapple fresh fruit $D$ ) was purchased from a local market.

Sampled juices were simply centrifuged, filtered and diluted 1:10 before chromatographic analyses (TLC and HPLC).

Juice from fresh pineapple was prepared after weighting and homogenizing of $100 \mathrm{~g}$ of pulp. $10 \mathrm{~g}$ of homogenized sample were taken and diluted in $100 \mathrm{~mL}$ of water. The solution was then centrifuged, filtered prior of the chromatographic analysis.

Samples analysed by Lane-Eynon method were previously filtered, decolorated by clarifying agents (by Carrez solutions) and diluted according to the following section.

\section{Titration Method}

The Lane-Eynon is a titration for determining the concentration of reducing sugars in a sample. It is well described in [13]. The carbohydrate solution, to be analyzed, is added by a burette to a flask containing a known amount of boiling copper sulfate solution (10 $\mathrm{mL}$ ) and a methylene blue indicator. The reducing sugars $(0.0515 \mathrm{~g}$ for $10 \mathrm{~mL}$ of fehling's solution) in the carbohydrate solution react with the copper sulfate present in the flask. Once all the copper sulfate in solution has reacted, any further addition of reducing sugars causes the indicator to change from blue to white. The volume of sugar solution required to reach the end point is recorded. The titration must be conducted at boiling point, very quickly and the samples to be analyzed have to be uncolored. $10 \mathrm{~mL}$ of solution ( $5 \mathrm{~mL}$ of Fehling's solution A copper sulfate +5 $\mathrm{mL}$ of Fehling's solution B sodium hydroxide and sodium potassium tartrate) are completely reduced by $0.05 \mathrm{~g}$ of reducing sugar, so that sample dilution is necessary when this proportion is not respected. Sucrose amount (not reducing sugar) is also determined by the Lane and Eynon method, but after the inversion of a portion of sample aqueous solution with chloridric acid followed by neutralization with alkali $(\mathrm{NaOH})$.

\section{Chromatographic Instrumentation}

TLC plates of silica gel $20 \mathrm{~cm} \times 20 \mathrm{~cm} x 250 \mu \mathrm{m}$ thickness were from Whatman (Carlo Erba) LC analysis was carried out by HPLC Jasco 880-PU coupled to RID detector WellChrom K-2301 Knauer
(Berlin Germany) and connected to an integrator HP mod. 3390A. The flow rate was set to $1 \mathrm{~mL} / \mathrm{min}$. The injector was a Rheodyne 8125 system with a $10 \mu \mathrm{L}$ sample loop. Chromatographic separations were performed on the following columns:

- $\quad \mathrm{CN}$ and $\mathrm{NH}_{2}$ columns $165 \mathrm{~mm}$ x 4,6 mm I.D., with particle size of $5 \mu \mathrm{m}$, packed in our lab (Kromasil and Spherisorb, Deeside Ind. Est. Queensferry, Cluey. U.K.).

- $\quad$ Luna Hilic column $150 \mathrm{~mm}$ x $4.6 \mathrm{~mm}$ I.D. with particle size of $5 \mu \mathrm{m}$, purchased by Phenomenex (Castel Maggiore-BO-Italy).

Several isocratic elutions were tried with methanol/ water and ACN/water, ethanol-water, isopropyl alcoholwater and methanol-acetonitrile-water.

\section{Quantitative Analysis}

The Lane-Eynon reaction is not precisely stoichiometric, which means that it should be necessary to prepare a calibration curve by carrying out the experiment with a series of standard solutions of known carbohydrate's concentration. Aqueous samples (triplicate, $n=3$ ) were previously filtered and discolored using clarifying agents (Carrez 1 and 2) to avoid interference due to the colored solution.

Dilution was done according to the method described in the section titration and several precautions, during the titration, were adopted according to the literature [14].

The per cent reducing sugar as glucose in the sample was determined in the first approximation as follows referring to $10 \mathrm{~mL}$ of Fehling's solutions:

\section{$\%$ of reducing sugar}

$(m / v)=\frac{0,0515 \times 100}{V m L \text { sample solution used in titration }} X$ dilution factor

$(V=$ volume $(\mathrm{mL})$ of sample aqueous solution used in titration; 0,0515= amount of glucose equivalent to 10 $m L$ Fehling's solutions).

The concentration of sucrose after inversion was calculated as difference between \% of total sugar after inversion and \% of reducing sugar multiplied by 0.95 (hydrolysis factor).

HPLC standard calibration curves were built with the external standard method (ESTD) by five injections of $10 \mu \mathrm{L}$ of six different standard solutions, containing 
Table 1: Standard Calibration Curve and LOD/LOQ of each Analyte

\begin{tabular}{|c|c|c|c|}
\hline Sugar & Standard calibration curve & $\mathbf{R}^{2}$ & LOD/LOQ (mg/mL) \\
\hline \hline fructose & $\mathrm{y}=1.22 \mathrm{E} 7( \pm 118929.4) \mathrm{x}+945398.8( \pm 609091.5)$ & 0,9999 & $0.1 / 0.3$ \\
\hline glucose & $\mathrm{y}=1.36 \mathrm{E} 7( \pm 170414.7) \mathrm{x}+1.4 \mathrm{E} 6( \pm 869295.6)$ & 0,9998 & $0.1 / 0.3$ \\
\hline sucrose & $\mathrm{y}=1,22 \mathrm{E} 7 \pm( \pm 96083.4) x+288343.6( \pm 982409.6)$ & 0,9999 & $0.2 / 0.4$ \\
\hline
\end{tabular}

analytes at increasing concentrations ranging from about $0-10 \mathrm{mg} \mathrm{mL}^{-1}$ (fructose and glucose) and 0-20 $\mathrm{mg} \mathrm{mL}^{-1}$ (sucrose). Working standard solutions were prepared diluting the stock solutions with water. Different calibration ranges were due to the expected concentration in real samples. The absolute analyte response (AREA) was plotted against the increasing analyte concentration to create the calibration curve, by applying the regression model to the calibration data set. The graphs were used to interpolate concentrations of the target analytes in real samples.

Good linearity was obtained in the investigated concentration range for each compound, as demonstrated by the correlation coefficients $\left(R^{2}\right.$ values 0.9999) of calibration curves, whose equations are given in Table 1. Instrumental detection limits $\left(\mathrm{LOD}_{\mathrm{s}}\right)$ was the concentration of injected analyte producing a peak with a signal-to-noise ratio $(\mathrm{S} / \mathrm{N})$ of 3 . These values were extrapolated from the $S / N$ of the respective peaks following an injection of the lowest standard concentration. The limit of quantification (LOQ) was estimated, in the same way, using the criterion of $(\mathrm{S} / \mathrm{N})$ of 6 . Table 1 shows the corresponding values in good agreement to other authors [1].

The precision was calculated by the relative standard deviation (RSD) for repeated measurements in the same day (intraday precision) and over three different days (inter day precision) injecting standard solution, at LOQ level, six times. The intraday precision $(n=6)$ was about $7 \%$ and the interday precision $(n=18) 10 \%$ for each analyte.

\section{RESULTS}

\section{TLC Analysis}

For a preliminary indication of the carbohydrate composition of commercial fruit juices a qualitative analysis by thin layer chromatography was performed. As shown in Figure 2 three different juices (pineapple, orange and apricot) were analyzed and their ratio front $\left(R_{\mathrm{f}}=\right.$ distance traveled by compound $\div$ distance traveled by solvent front) compared to those of three standard solutions of sucrose, fructose and glucose $(1 \%)$. To avoid tailing effect, the juices were diluted in water 1:10 before applying on the silica gel. Several eluting mobile phase were tried and the best compromise was ethyl acetate/ isopropyl alcohol /water $=65: 3: 5$.

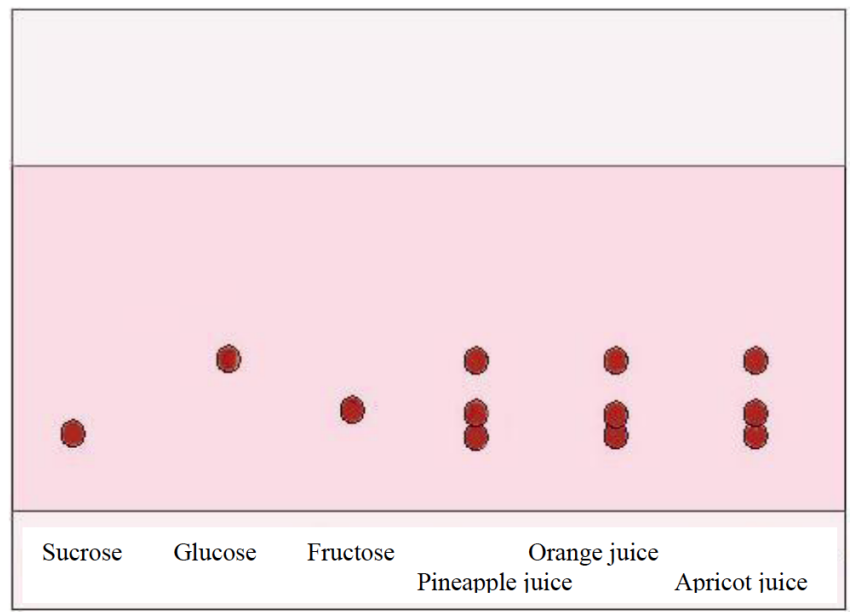

Figure 2: Preliminary TLC analysis of three manufacturer's commercial juices (diluted 1:10) compared to sucrose, glucose and fructose standard solution (1\%). Glass plates 20 $\mathrm{cm} \times 20 \mathrm{~cm}$ precoated with $0.25 \mathrm{~mm}$ dry silica gel.

Mobile phase ethyl acetate/ isopropyl alcohol /water $=65: 3$ : 5 .

Spraying detection agent was made from a mixture of anisaldeide $(1 \mathrm{~mL})$ added to $100 \mathrm{~mL}$ of glacial acetic acid containing $2 \mathrm{~mL}$ of concentraded sulphuric acid (96\%).

Spraying detection agent was freshly made from a mixture of anisaldeide $(1 \mathrm{~mL})$ added to $100 \mathrm{~mL}$ of glacial acetic acid containing $2 \mathrm{~mL}$ of concentrated sulphuric acid (96\%). After spraying, the silica gel plate was left for at least $30 \mathrm{~min}$ at $100{ }^{\circ} \mathrm{C}$ in oven.

Although TLC is not a sensitive technique, it was able to confirm the presence of sugars in diluted juices by dark stains on pink bottom.

\section{Refractometric Measurements}

For a preliminary indication of the amount of total carbohydrates in juices, measurements were performed on a sugar Brix refractometer, measuring the percent Brix, or relative sugar concentration, of a 
Table 2: Content of Fructose, Glucose and Sucrose in Three Commercial Packaged Fruit Juices and in a Fresh HomeMade Juice. Comparison between HPLC, Lane Eynon Titration, Brix Concentration and Manufacturer's Label

\begin{tabular}{|c|c|c|c|c|c|}
\hline Sample & Carbohydrates & $\begin{array}{c}\text { concentration by } \\
\text { HPLC } \\
(\mathrm{g} / 100 \mathrm{~mL})\end{array}$ & $\begin{array}{c}\text { concentration by } \\
\text { Lane-Eynon titration } \\
(\mathrm{g} / 100 \mathrm{~mL})\end{array}$ & Brix Concentration & $\begin{array}{c}\text { manufacturer's } \\
\text { label } \\
(\mathrm{g} / 100 \mathrm{~mL})\end{array}$ \\
\hline \multirow{4}{*}{$\begin{array}{c}\text { Brand } 1 \\
\text { Commercial } \\
\text { Apricot juice } \\
\left(^{*}\right)\end{array}$} & Fructose $(F)$ & $5.30 \pm 0.07$ & $(F+G)$ & \multirow{3}{*}{$(F+G+S)$} & \\
\hline & Glucose (G) & $4.50 \pm 0.09$ & $8.0 \pm 0.1$ & & \\
\hline & Sucrose (S) & $6.00 \pm 0.11$ & $7.6 \pm 0.1$ & & \\
\hline & Total $(\mathbf{F + G + S})$ & $15.80 \pm 0.16$ & $15.6 \pm 0.1$ & $17.0+0.5$ & 16.1 \\
\hline \multirow{4}{*}{$\begin{array}{c}\text { Brand } 2 \\
\text { Commercial } \\
\text { Orange juice } \\
\qquad\left(^{* *}\right)\end{array}$} & Fructose & $3.40 \pm 0.05$ & $(F+G)$ & \multirow{3}{*}{$(F+G+S)$} & \\
\hline & Glucose & $3.30 \pm 0.07$ & $6.5 \pm 0.1$ & & \\
\hline & Sucrose & $3.10 \pm 0.09$ & $3.2 \pm 0.2$ & & \\
\hline & Total $(\mathbf{F + G + S})$ & $9.80 \pm 0.12$ & $9.7 \pm 0.2$ & $10.5 \pm 0.5$ & 9.0 \\
\hline \multirow{4}{*}{$\begin{array}{c}\text { Brand } 3 \\
\text { Commercial } \\
\text { Pineapple } \\
\text { juice } \\
\left({ }^{* \star}\right)\end{array}$} & Fructose & $3.60 \pm 0.06$ & $(F+G)$ & \multirow{3}{*}{$(F+G+S)$} & \\
\hline & Glucose & $4.00 \pm 0.11$ & $7.3 \pm 0.1$ & & \\
\hline & Sucrose & $4.10 \pm 0.09$ & $4.4 \pm 0.1$ & & \\
\hline & Total $(\mathbf{F + G + S})$ & $11.70 \pm 0.15$ & $11.7 \pm 0.1$ & $12 \pm 0.5$ & 11.0 \\
\hline \multirow{4}{*}{$\begin{array}{l}\text { Fresh pineapple } \\
\text { juice }\end{array}$} & Fructose & $1.55 \pm 0.01$ & $(F+G)$ & \multirow{3}{*}{$(F+G+S)$} & \\
\hline & Glucose & $0.27 \pm 0.04$ & $2.0 \pm 0.2$ & & \\
\hline & Sucrose & $6.25 \pm 0.08$ & $6.2 \pm 0.2$ & & \\
\hline & Total $(\mathbf{F + G + S )}$ & $8.07 \pm 0.06$ & $8.2 \pm 0.2$ & $9 \pm 0.5$ & \\
\hline
\end{tabular}

$\left({ }^{*}\right)=$ juice added by sucrose and glucose/fructose syrup.

$\left({ }^{\star *}\right)=$ juice without added sugar.

liquid sample. Operational theory for refractometry is presented in [15]. Sugar content concentrations in Brix are shown for the investigated samples in Table 2. The findings indicate that the values for the total sugar concentration usually are a bit higher than declared in the label. The measurement is quick and simple to carry out, can be performed with simple hand-held instruments and does not require any preventive manipulation of the samples [16].

\section{HPLC Analysis of Carbohydrates}

The separation of sugars is a challenge for the food technologists, because they are extremely hydrophilic and highly polar compounds, uncharged at neutral $\mathrm{pH}$ and do not have a UV chromophore. HPLC is currently the most important chromatographic method for analyzing carbohydrates because it is capable of rapid, specific, sensitive and precise measurements. A good separation of carbohydrates, on the basis of their partition coefficients, polarities or sizes, depends on the type of column and mobile phase. Several experiments were performed to select the most suitable column for our purpose. Five columns with different stationary phases, length and diameters were tried, in particular: $\mathrm{CN}, \mathrm{C}_{18}, \mathrm{C}_{8}$, and HILIC NH $\mathrm{NH}_{2}$ and silica. As noticed from other authors [10] traditional reversed phase columns cannot be used for underivatized sugars, as the stationary phase does not provide the required retention. Selectivity of the HILIC is substantially different from $\mathrm{C}_{18}, \mathrm{C}_{8}$ and $\mathrm{CN}$ columns, making it ideal for difficult polar samples such as the analysis of monoand disaccharides. Hdrophilic interaction chromatography (HILIC), using a high polar packing material, separation is based on the hydrophilic interactions of the sugars on the stationary phase typically using an eluent with a majority of polar organic solvent.

Silica and amino HILIC columns were tried and amino functional groups were considered the best, owning a better separation [17]. 
A refractive index detector (RID) was used for the detection. Refractive index was measured at boundary with quartz. The RI is dependent by temperature and mobile phase and so the measurements were made at a specific temperature $\left(20^{\circ} \mathrm{C}\right)$, waiting until the chromatographic system was stable and under isocratic conditions. Acetonitrile-water is the typical mobile phase, but also other combination of solvents were tried (methanol-water, ethanol-water, isopropyl alcohol-water and methanol-acetonitrile-water). In this case the analysis was carried out in isocratic, using normal-phase mode of separation, with acetonitrilewater=90: 10, and the peaks were well resolved in 16 minutes. Figure 3 shows the separation of $10 \mu \mathrm{L}$ of a standard solution $(5 \mathrm{mg} / \mathrm{mL}$ fructose and glucose and $10 \mathrm{mg} / \mathrm{mL}$ sucrose) containing the three analytes fructose, glucose and sucrose eluted respectively at 6.80, 8.15 and $16.30 \mathrm{~min}$. Figures 4A, 4B, 4C and 4D show the chromatographic profile of four real samples: three juices commercially manufactured (apricot $A$, orange $B$, pineapple $C$ ) and one sample freshly made (fresh pineapple juice D). To qualitative purposes, the correspondence of retention times was used for the identifications. A visual comparison of chromatograms shows immediately that there aren't great differences in the chromatographic profiles between sample B and C, whereas in sample $A$ there is a more significant

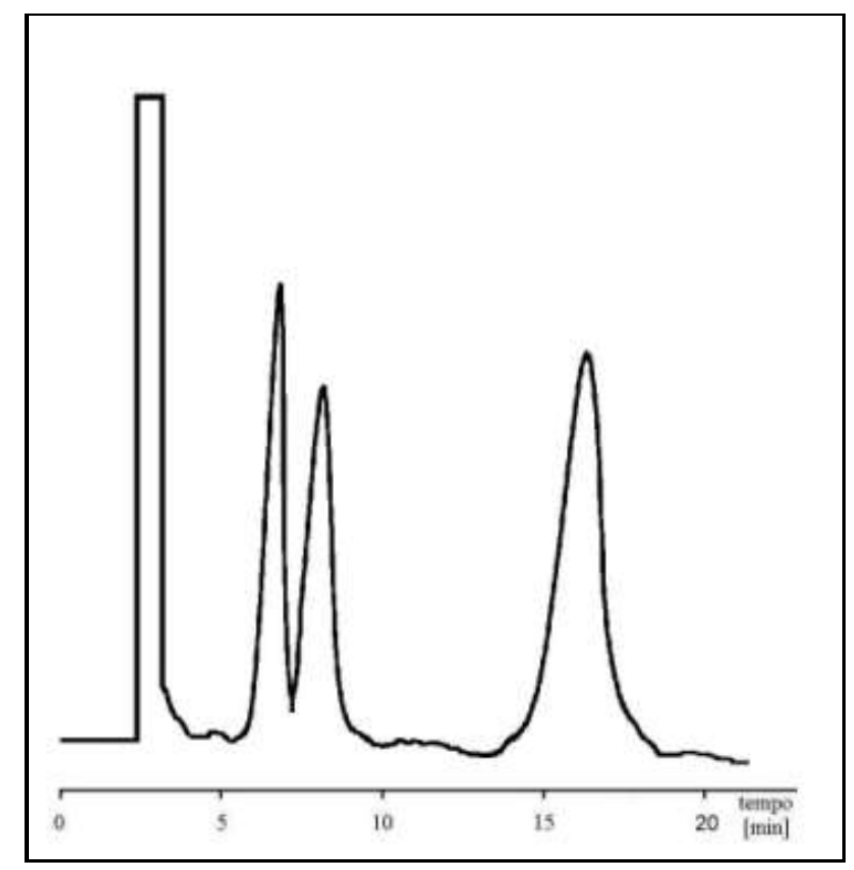

Figure 3: HPLC-RID analysis of $10 \mu \mathrm{L}$ of standard mixture of fructose, glucose $(5 \mathrm{mg} / \mathrm{mL})$ and sucrose $(10 \mathrm{mg} / \mathrm{mL})$. Column packed in our lab propyl- $\mathrm{NH}_{2}$ (I.D $4.6 \mathrm{~mm} \times 110 \mathrm{~mm}$ length $5 \mu \mathrm{m}$ particle size).

Flow rate $1 \mathrm{~mL} / \mathrm{min}$. Mobile phase: acetonitril-water= 90:10 $\mathrm{v} / \mathrm{v}$ in isocratic condition. presence of sugars as declared in the label from the producer. Pineapple chromatograms $C$ and $D$ show immediately different relative ratios between sugars in commercial and fresh pineapple juice.

As the samples were directly injected to the chromatograph after a simple filtration and dilution $(1: 10)$ without particular manipulation, the recovery of the method was considered $100 \%$.

Table 2 summarizes in the second column the quantitative results obtained by HPLC analysis on real samples, using the standard calibration curves of Table 1. Sugar concentrations in real samples were sensibly bigger than the LOD and LOQ value (shown in Table 1). The ratio between fructose and glucose is around 1 with the exception of fresh pineapple. The importance of this ratio has been well investigated by Walker RW et al. [18], since an excessive fructose consumption is hypothesized to be associated with risk of metabolic disease.

\section{Lane-Eynon Titration of Carbohydrates}

As known this method is affected by several limits. First of all the titration must be rapid and under controlled temperature and depends on the reagent concentrations; it can be susceptible to interference from other types of reducing agents, cannot distinguish between different types of reducing sugar, and it cannot determine the concentration of non-reducing sugars. The reaction is not precisely stoichiometric, that means that formulas reported previously are only an approximation. On the other hand it is very simple, cheap, and quite fast to perform.

Table 2, shows the results obtained applying formulas reported before in the experimental part. Reducing sugars are given as sum of glucose and fructose, whereas sucrose, being a not reducing sugar, is obtained indirectly by the difference between total sugar, after chemical hydrolysis of the samples, and reducing sugar calculated before corrected by 0.95 (hydrolysis factor).

\section{DISCUSSION}

As told before, the TLC and the refractometric measures were performed to have a very fast, and an indicative qualitativelquantitative response. To use sugar content in Brix more rigorously it would be necessary to build a calibration curve with sugar standard solutions and at least to centrifuge or filter the juices so as to remove the pulp and repeat the 


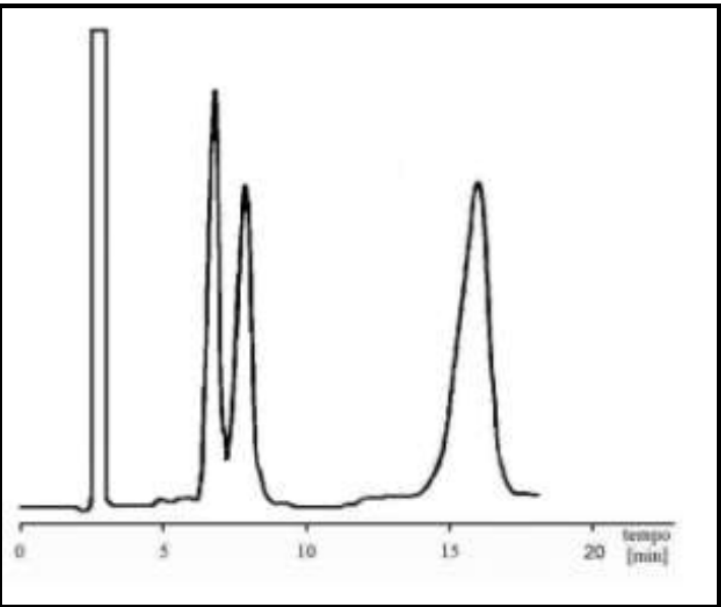

(A)

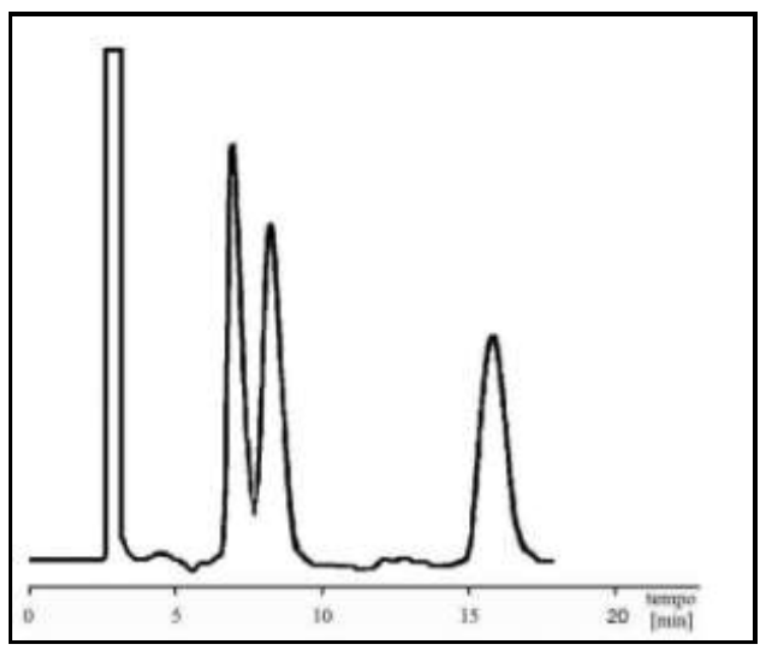

(C)

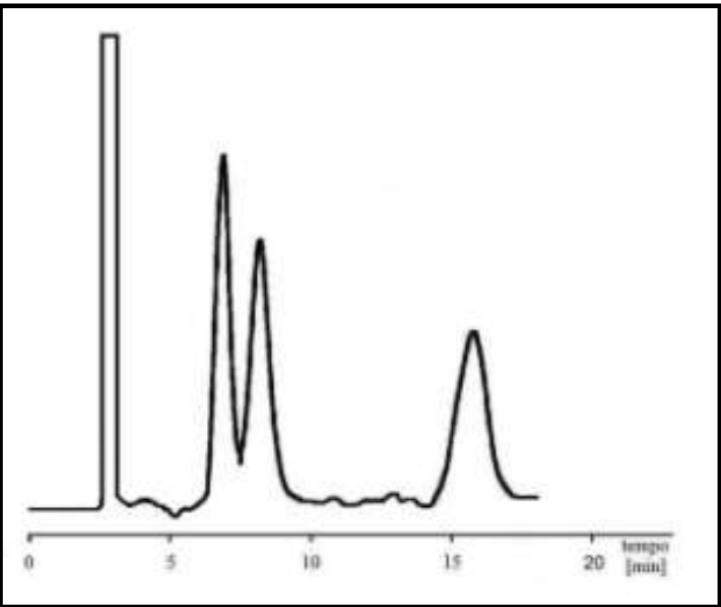

(B)

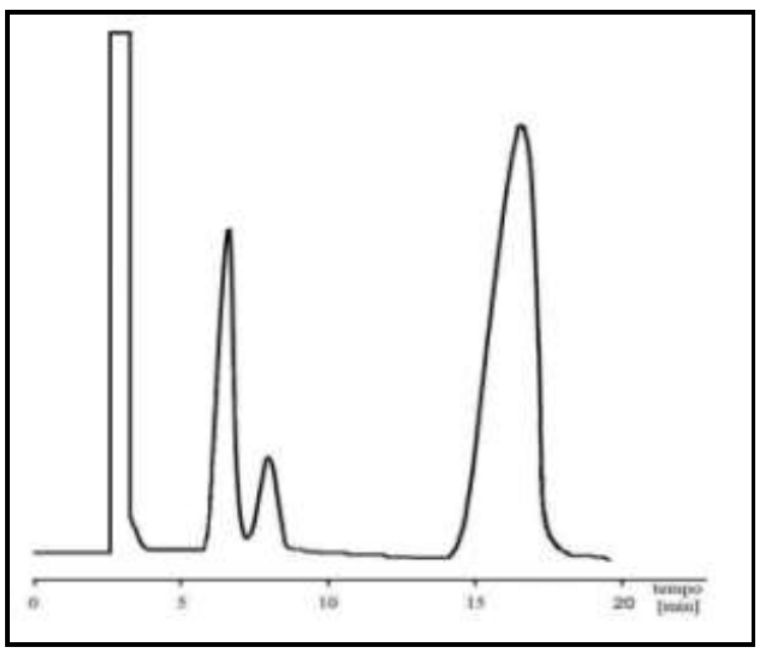

(D)

Figure 4: HPLC-RID analysis of $10 \mu \mathrm{L}$ of commercial juices of: apricot A), orange B), pineapple $\mathbf{C}$ ) and of fresh juice of pineapple D). Other conditions as in Figure 3.

The samples were prepared as described in the text.

measures on the surnatant. In this case the values seem to be a bit overestimated and the method provides the total amount of sugar and does not give indication on the single components.

HPLC has largely replaced the earlier methods of thin-layer chromatography (TLC) for the determination of mono- and disaccharides due to its advantages with regard to separation efficiencies [19]. The HPLC method distinguishes itself as a fast and accurate way of obtaining the amount of different sugars, with direct injection of a sample with little or without pretreatment and simple interpretation of chromatograms.

The Lane and Eynon method is used to determine the reducing sugars content in food and beverages, whereas sucrose measurement is indirect.
To assess the reliability of these last assays, we calculated the coefficient of variation for the repeated measurements. The volumetric and chromatographic analyses, relatively to the same sample, were done the same day, being sugars fermentable.

The trends and the comparison between the two methods in the four different samples are presented as histograms in Figure 5A, 5B, 5C and 5D. Different colors are used for sucrose (S) and for the sum of sugars glucose+ fructose $(\mathrm{G}+\mathrm{F})$. As seen, usually the two methods are in good agreement, in particular for the total content of sugars. In addition, the total sugar content of the beverages, as assessed by the laboratory, ranged between 90 and $110 \%$ with respect to what listed on the food label, but it's not known how the producers calculate them. 


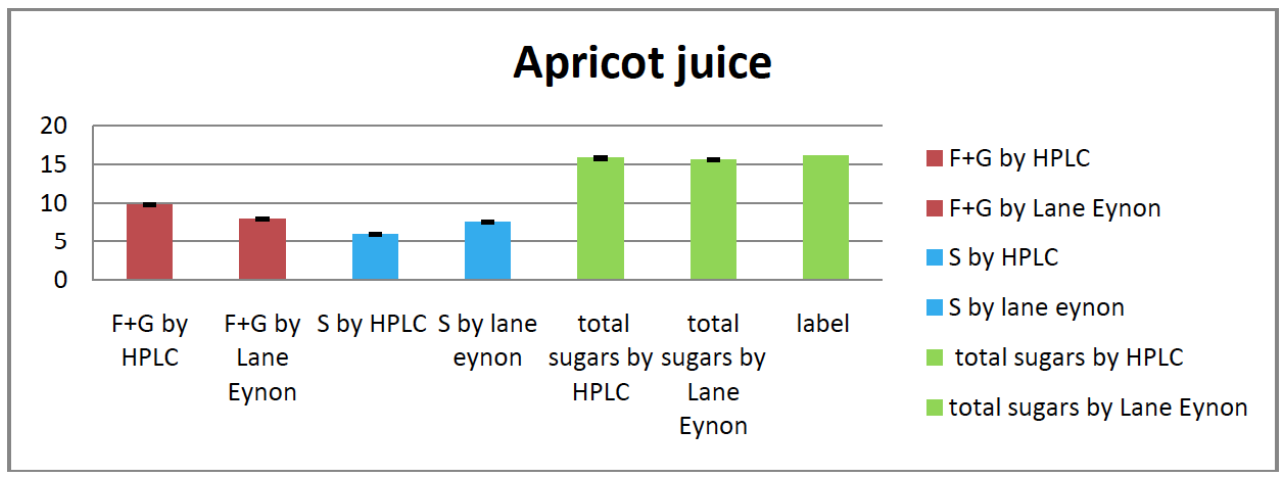

(A)

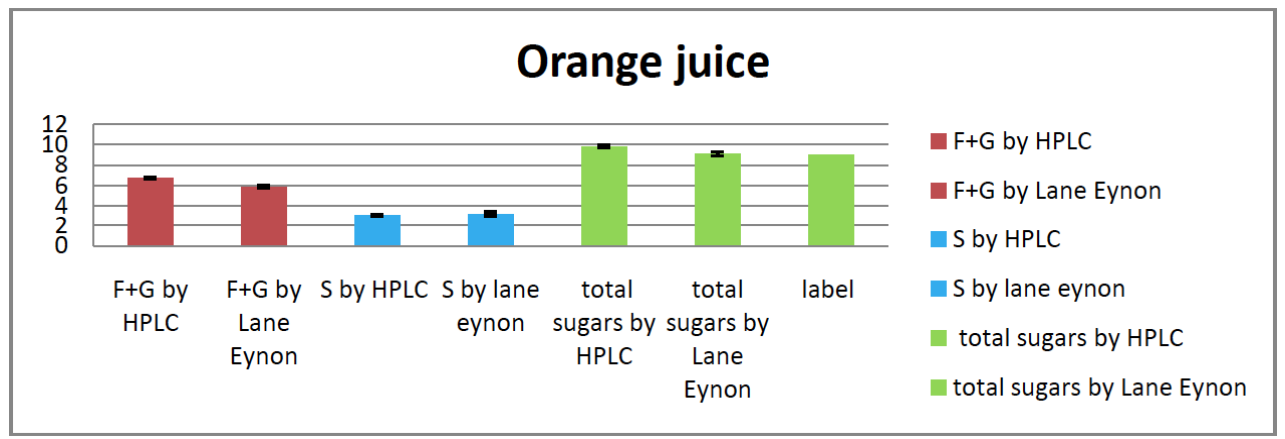

(B)

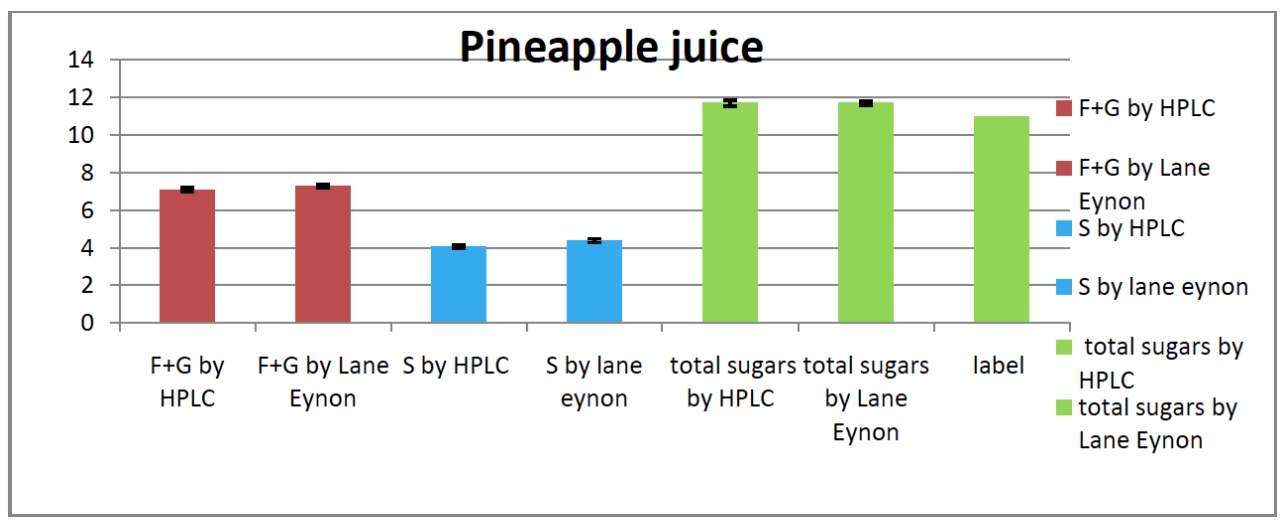

(C)

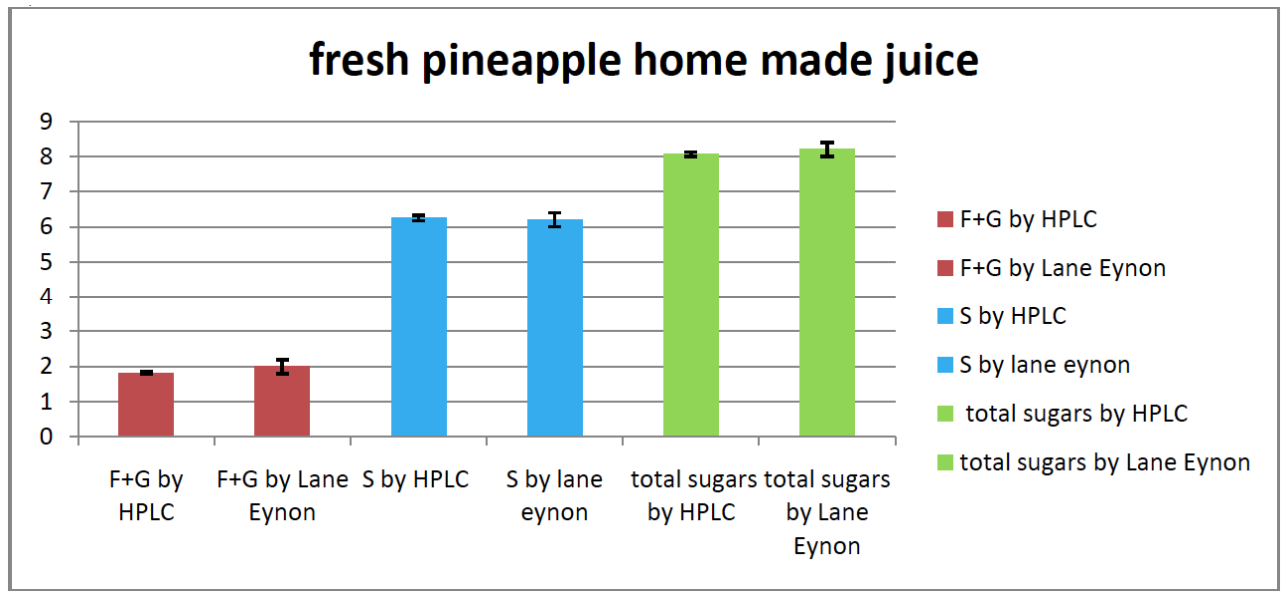

(D)

Figure 5: Comparison between HPLC and Lane-Eynon results in A) apricot juice (marketed) B) orange juice (marketed) C) pineapple juice (marketed) and D) fresh pineapple juice. 
The slight differences between the content of $\mathrm{G}+\mathrm{F}$ and $S$ with the two methods (in particular in apricot juice) could be due both to higher manipulation of samples before volumetric analysis and both to the need of standardization of Lane Eynon titration. Sample A in particular is made from puree and juice and this can introduce inaccuracy during clarifying operations. Besides, usual cautions must be taken to avoid errors on the percentage of reducing sugars due to the influence of rate of boiling solution, and to the preparation of mixed Fehling's solution $\left(10 \mathrm{~cm}^{3} \mathrm{~A}+10\right.$ $\mathrm{cm}^{3} \mathrm{~B}$ or $20 \mathrm{~cm}^{3} \mathrm{~A}$ and $\mathrm{B}$ ). On the other hand the sucrose dosage is made indirectly after chemical hydrolysis.

On the other side comparison between fresh homemade and commercial pineapple juices revealed a great discrepancy between the trend of the sugars by HPLC. We were expecting in marketed pineapple juice (being a concentrated juice with no sugar added) the same profile of fresh fruit, but in the last one sucrose is the most abundant sugar, more than the double, followed by fructose and glucose. Although our data on fresh fruit are aligned to those of other authors [20], and experiments were repeated on three different aliquot of the same fruit, other test should be done on different pineapple fruits before giving definitive conclusions.

\section{CONCLUSIONS}

In the current literature there are a lot of papers about sugar composition in food and beverages with different analytical methods, but very few about the comparison between them on the same sample.

In this paper different analytical approaches of sugar measurement on fruit juices were compared.

HPLC, considering the lack of manipulation of the samples before the analysis resulted the simplest and fastest. As known, it gives qualitative/quantitative information on the single carbohydrate. Results obtained by HPLC were in good agreement with those obtained by Lane-Eynon titration on the same samples, showing the robustness and the accuracy of the techniques. Sugar content issued by the lab was aligned to that declared in the label. Apricot juice with added sugar didn't show any particular difference in the sugar content with respect to the other two juices.

The most surprising results were the inconsistency between sugar profile and relative sugar amounts in fresh pineapple and marketed juice with no sugar added. This is an important and delicate topic to be deeply studied, and more investigation are mandatory before drawing conclusions.

\section{REFERENCES}

[1] Petkova NT, Brabant PA, Masson A, Denev P. HPLC analysis of mono and disaccharides in food products. Conference paper of Food Science, Engineering and technology 2013; Oct 18-19 At Plovdiv, Bulgaria, Volume LX

[2] Zhang Q, Hvizd M, Bailey B, Thomas D, Plante M, Acworth I. Carbohydrate Analysis in Beverages and Foods Using Pulsed Amperometric Detection or Charged Aerosol Detection. In Thermo Scientific Poster Note • PN71433-EN 1114S.

[3] Ball S, Mapp K, Lloyd L. Sugar Analysis: An aqueous alternative to the use of amino-bonded HPLC columns with acetonitrile eluents. www.varianinc.com. Available from: http://www.agilent.com/cs/library/posters/public/SI2576\%20HPLC $\% 202009 \% 20$ sugar\%20analysis, $\% 20$ no $\% 20$ ACN.pdf.

[4] Birch GG. Methods of carbohydrate analysis. Asean Journal on science and technology for development 1985; 2(1):8897.

[5] AOAC 923.09 Invert Sugar in Sugar and Sirups pag 10161017 in Official methods of analysis of the Association of Official Analytical Chemists (AOAC). Food composition; additives; natural contaminats vol $215^{\text {th }}$ edition 1990 Edited by Kenneth Helrich.

[6] Ruiz-Matute $\mathrm{Al}$, Hernández-Hernández $\mathrm{O}$, RodríguezSánchez S, Sanz ML, Martínez-Castro I. Derivatization of carbohydrates for GC and GC-MS analyses. J Chromatogr B Analyt Technol Biomed Life Sci 2011; 879(17-18): 1226-40. http://dx.doi.org/10.1016/j.jchromb.2010.11.013

[7] Pomata D, Di Filippo P, Riccardi C, Buiarelli F, Gallo V. Determination of non-certified levoglucosan, sugar polyols and ergosterol in NIST Standard Reference Material. Atmospheric Environment 2014; 84: 332-338. http://dx.doi.org/10.1016/j.atmosenv.2013.11.069

[8] Augustin MA, Khor KL. Determination of Sugars in Soft Drinks by High Performance Liquid Chromatography. Pertawka 1986; 9(1): 119 -123.

[9] Rajakyla E, Paloposki M. Determination of sugars (and betaine) in molasses by High Performance Liquid chromatography. Comparison of the results with those obtained by the Classical Lane-Mynon method. J of Chromatogr 1983; 282: 595-602. http://dx.doi.org/10.1016/S0021-9673(00)91636-4

[10] Salman M, Abdel-Hameed E-S S, Bazaida S A, AlShamranib M G, Mohamed H F. Liquid chromatographymass spectrometry (LC-MS) method for the determination of sugars in fresh pomegranate fruit juices. Der Pharma Chemica 2014; 6(5): 320-333.

[11] Folkes DJ, Jordan MA. Mono- and Disaccharides: Analytical Aspects. In: Eliasson AC Editor. Carbohydrates in food. 2nd ed. London CRC Press Taylor \& Francis Group, LLC 2006; pp. 2-40.

http://dx.doi.org/10.1201/9781420015058.ch1

[12] Buiarelli F, Canepari S, Di Filippo P, Perrino C, Pomata D, Riccardi C, Speziale R. Extraction and analysis of fungal spore biomarkers in atmospheric bioaerosol by HPLC-MSMS and GC-MS. Talanta 2013; 105: 142-151. http://dx.doi.org/10.1016/j.talanta.2012.11.006

[13] Glazer Peres K, Teixeira O C, Peres MA, dos Santos Raymundo M, Fett R. Sugar content in liquid oral medicines for children. Rev Saude Publica 2005; 39(3): 486-489. http://dx.doi.org/10.1590/S0034-89102005000300022 
[14] Dunsmore A, Mellet P, Wolff $M$. Some factors affecting the Lane and Eynon titration method for determining reducing sugars in sugar products. In: Proceedings of The South African Sugar Technologists' Association - June 1980.

[15] Serpen JY. Comparison of Sugar Content in bottled $100 \%$ fruit juice versus extracted juice of fresh fruit. Food and nutrition science (FNS) 2012; 3: 1509-1513. http://dx.doi.org/10.4236/fns.2012.311196

[16] Ramasami P, Jhaumeer-Laullo $S$, Rondeau $P$, Cadet $F$, Seepujak H, Seeruttun A. Quantification of Sugars in Soft Drinks and Fruit Juices by Density, Refractometry, Infrared Spectroscopy and Statistical Methods. S Afr J Chem 2004; 57: 24-27.

[17] Showa Denko KK, Turcotte M, Showa Denko A, Naoya N. New HILIC Column for Saccharide Analysis in The application notebook LC GC. 2014; sept. Available from: http://www.chromatographyonline.com/new-hilic-columnsaccharide-analysis
[18] Walker RW, Dumke KA, Goran MI. Fructose content in popular beverages made with and without high-fructose corn syrup. Nutrition 2014; 30: 928-935. http://dx.doi.org/10.1016/j.nut.2014.04.003

[19] Krobards K, Whitelaw M. Chromatography of monosaccharides and disaccharides. J of Chromatog 1986; 373: 81-110.

http://dx.doi.org/10.1016/S0021-9673(00)80209-5

[20] Cordenunsi B, Saura-Calixtm F, Diaz-Rubio M E, Zuleta A Tiné M A, Buckeridge M S, da Silva G B, Carpio C, Giuntini B $\mathrm{E}$, de Menezes E W, Lajolo F. Carbohydrate composition of ripe pineapple (cv. perola)and the glycemic response in humans. Ciênc Tecnol Aliment 2010; 30(1): 282-288. http://dx.doi.org/10.1590/S0101-20612010000100041 